\title{
Crosstalk between Adipocytes and Immune Cells in Adipose Tissue Inflammation and Metabolic Dysregulation in Obesity
}

\author{
Jin Young Huh, Yoon Jeong Park', Mira Ham, and Jae Bum Kim*
}

\begin{abstract}
Recent findings, notably on adipokines and adipose tissue inflammation, have revised the concept of adipose tissues being a mere storage depot for body energy. Instead, adipose tissues are emerging as endocrine and immunologically active organs with multiple effects on the regulation of systemic energy homeostasis. Notably, compared with other metabolic organs such as liver and muscle, various inflammatory responses are dynamically regulated in adipose tissues and most of the immune cells in adipose tissues are involved in obesity-mediated metabolic complications, including insulin resistance. Here, we summarize recent findings on the key roles of innate (neutrophils, macrophages, mast cells, eosinophils) and adaptive (regulatory T cells, type 1 helper T cells, CD8 T cells, B cells) immune cells in adipose tissue inflammation and metabolic dysregulation in obesity. In particular, the roles of natural killer T cells, one type of innate lymphocyte, in adipose tissue inflammation will be discussed. Finally, a new role of adipocytes as antigen presenting cells to modulate $\mathbf{T}$ cell activity and subsequent adipose tissue inflammation will be proposed.
\end{abstract}

\section{ADIPOSE TISSUE INFLAMMATION AS AN IMPORTANT MEDIATOR OF INSULIN RESISTANCE IN OBESITY}

The prevalence of obesity and overweight continues to rise, contributing increasingly to morbidity and mortality. In particular, obesity is one of the key factors for the development of metabolic diseases such as hypertension, hyperlipidemia, atherosclerosis, and type 2 diabetes (Moller and Kaufman, 2005). Recent findings have demonstrated that obesity is tightly associated with systemic chronic inflammation. There is accumulating evidence that cytokine production by expanded adipose

School of Biological Science, Institute of Molecular Biology and Genetics, ${ }^{1}$ Department of Biophysics and Chemical Biology, Seoul National University, Seoul 151-742, Korea

*Correspondence: jaebkim@snu.ac.kr

Received 1 April, 2014; accepted 4 April, 2014; published online 30 April, 2014

Keywords: adipocyte, adipose tissue inflammation, antigen presentation, CD1d, CD4 T cell, CD8 T cell, invariant natural killer T cell, macrophage, obesity tissue results in elevated serum levels of inflammatory cytokines such as tumor necrosis factor (TNF)- $\alpha$ and interleukin (IL) -6 in obese individuals (Hotamisligil et al., 1993; Xu et al., 2003). Subsequently, the elevated inflammatory stimuli induce the activation of IKK $\beta / N F \kappa B$ and JNK pathways, which negatively regulate insulin action in adipocytes and hepatocytes (Shoelson et al., 2006). Therefore, it has been proposed that accumulated inflammatory responses in adipose tissue may play a causal role in obesity-induced insulin resistance. Of note, it has been reported that in the early stages of obesity inflammatory gene expression is selectively induced in adipose tissues, but not in other metabolic organs including liver and skeletal muscle (Lee et al., 2011). Thus, adipose tissues appear to act as priming tissues that respond to a high-fat diet (HFD) and initiate inflammation in obesity. Understanding the inflammatory responses in adipose tissues of obese individuals is therefore clearly of clinical importance.

Adipose tissue is composed of various cell types including adipocytes, fibroblasts, endothelial cells, and various immune cells. Immune cells within adipose tissue are largely categorized into innate and adaptive immune cells. Innate immune cells include macrophages, neutrophils, eosinophils, and mast cells, whereas adaptive immune cells include various subtypes of $T$ cells and $B$ cells. Furthermore, natural killer $T$ cells, one type of innate lymphocyte, were recently reported as regulators of adipose tissue inflammation in obesity. In lean adipose tissue, various anti-inflammatory immune cells such as M2 type macrophages, regulatory $\mathrm{T}$ cells, and eosinophils are engaged in the maintenance of insulin sensitivity. However, during progression of obesity, changes in the number and activity of various immune cells play prominent roles in the progression of insulin resistance via production of pro-inflammatory mediators (Fig. 1). Moreover, recent findings have suggested that adipocytes corroborate with certain immune cells and directly regulate the activation and proliferation of adipose immune cells. The purpose of this review is to summarize current understanding of the roles of innate and adaptive immune cells in adipose tissue inflammation and insulin resistance in obesity. In addition, the new role of adipocytes as antigen presenting cells for $T$ cells will be discussed.

\section{MACROPHAGES}

Macrophages, tissue-resident phagocytes, perform various roles 


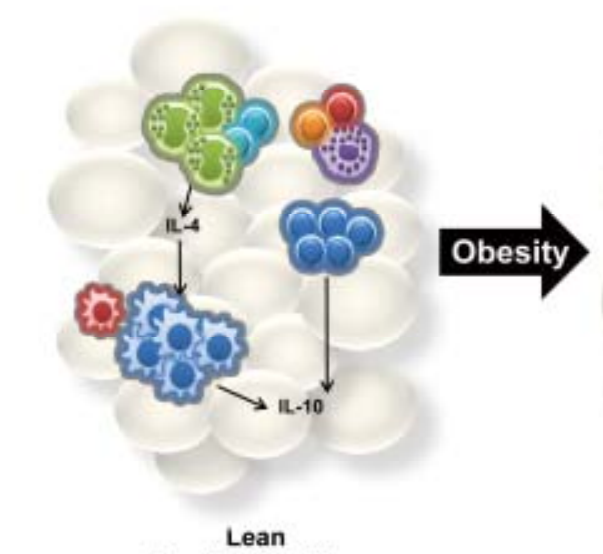

Insulin sensitive

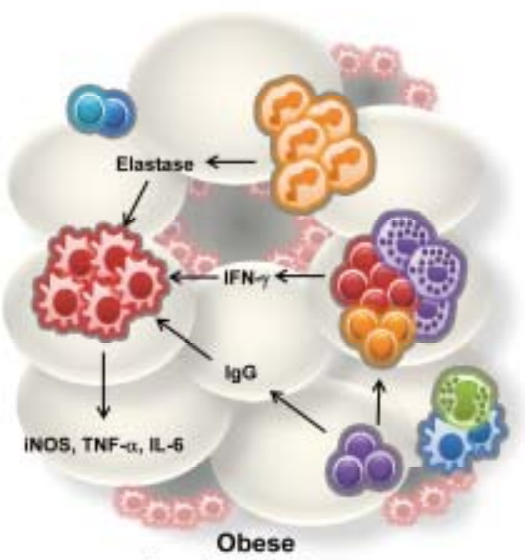

Insulin resistance

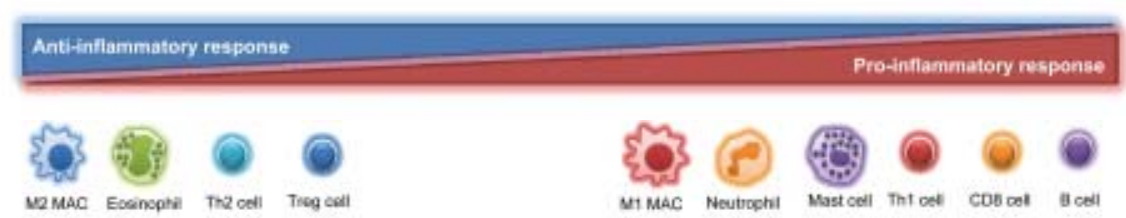

Fig. 1. Interactions of adipose tissue immune cells. In lean adipose tissue, IL4 secreted by eosinophils and Th2 cells activates M2 type macrophages, which express arginase and anti-inflammatory cytokines such as IL-10. Regulatory T (Treg) cells also play an important role in anti-inflammatory responses via cell-cell contact or cytokine secretion involving IL-10. However, in obese adipose tissue the number of pro-inflammatory immune cells is increased and that of antiinflammatory immune cells is decreased. Neutrophils, which are early responders to inflammatory responses, infiltrate the adipose tissue where they secrete elastase and also stimulate M1 type macrophage infiltration and pro-inflammatory cytokine secretion. In addition, levels of IFN- $\gamma$-secreting cell types, such as Th1 cells, CD8 T cells, and mast cells, are elevated in obese adipose tissue. B cells also play a pro-inflammatory role through secretion of obesity-induced lgG. including scavenging cellular debris derived from apoptotic cells, regulating angiogenesis, and remodeling the extracellular matrix (Chawla et al., 2011). Although macrophages comprise 10$15 \%$ of stromal vascular cells (SVCs) in visceral adipose tissues (VAT) of lean subjects, their numbers are increased to 40$50 \%$ of the SVCs of VAT in obese humans and mouse models (Weisberg et al., 2003). Monocytes are differentiated into classically activated macrophages (M1) or alternatively activated macrophages (M2) upon stimulation. The major populations of adipose tissue macrophages (ATMs) that reside in lean adipose tissue are different from those residing in obese adipose tissues. For example, in the lean status, the predominant ATM population is M2 macrophages, which express high levels of arginase-1, the mannose receptor (CD206), and CD301 and secrete anti-inflammatory cytokines including IL-10 and IL-1 receptor antagonist (IL-1Ra). Th2 type cytokines such as IL-4, IL-10, and IL-13 stimulate the M2 polarization (Chawla et al., 2011; Lumeng et al., 2007a). In contrast, in obesity, interferon (IFN)- $\gamma$ and lipopolysaccharide (LPS) drive polarization of recruited monocytes toward classically activated M1 type macrophages and promote the secretion of pro-inflammatory cytokines such as TNF- $\alpha$, IL-6, IL-1 $\beta$, IL-12, and MCP-1 (Ham et al., 2013; Mathis, 2013). One of the key characteristics of M1 macrophages is the surface expression of CD11c proteins in addition to macrophage-specific markers such as F4/80 and CD11b. Previous studies have demonstrated that the major population of infiltrated M1 macrophages in adipose tissue originates from circulating monocytes in the blood (Lumeng et al., 2007b). Interaction between MCP-1 and CCR2 appears to be crucial for obesity-induced macrophage infiltration into adipose tissue. Very recently, it has been reported that proliferation of local macrophages also contributes to increased adipose tissue inflammation (Amano et al., 2014). Compared with M2 macrophages, accumulation of pro-inflammatory M1 macrophages in adipose tissue provokes whole body insulin resistance. For example, ablation of CD11c-positive cells leads to marked augmentation of insulin sensitivity, accompanied by diminished inflammatory responses including macrophage infiltration and inflammatory cytokine gene expression in adipose tissue and lower levels of serum inflammatory cytokines (Patsouris et al., 2008). However, we cannot exclude the possibility that the roles of M1 macrophages demonstrated in a CD11c knockout (KO) mouse model might also be attributed to dendritic cells because CD11c is one of the pan markers of dendritic cells.

\section{NEUTROPHILS}

Neutrophils are the most abundant white blood cells (WBCs) in the immune system. Since neutrophils are short-lived cells and are rapidly recruited to infected tissues, they are well known as a primary effector cell type in acute inflammatory responses (Pham, 2006). Obese patients exhibit significant increases in both neutrophil-derived proteins, including myeloperoxidase and calprotectin, and the expression of markers for neutrophi activation such as CD66b (Nijhuis et al., 2009). Talukdar et al. (2012) have demonstrated that neutrophil infiltration is elevated in adipose tissue of mice fed a HFD over a short term (3 days). In addition, both neutrophil elastase KO mice and mice treated with elastase inhibitor have reduced adipose tissue inflammation and improved glucose tolerance (Talukdar et al., 2012). Therefore, it has been suggested that neutrophils are implicated in the modulation of adipose tissue inflammation in the early stage of obesity.

\section{MAST CELLS}

Mast cells are mainly localized in connective and mucosal tissues where they act as first line responders against invading pathogens (Bischoff, 2007). Mast cells play critical roles in allergy and anaphylaxis. However, recent studies have indicated that mast cells might also regulate adipose tissue inflammation in obesity. Mast cells contain many granules loaded with various mediators including histamine, serotonin, heparin, serine protease, eicosanoids, and cytokines. Upon receiving stimulatory signals, they rapidly release these mediators to regulate inflammatory responses. Interestingly, it has been reported that 
the serum level of mast cell-derived tryptase and the number of adipose tissue mast cells are increased in obese patients and HFD-fed mice, respectively (Liu et al., 2009). Additionally, mice lacking mast cells are resistant to diet-induced obesity (DIO) and show reduced inflammatory responses and insulin resistance. Among various mediators produced by mast cells, IL- 6 and IFN- $\gamma$ lay a major role in these positive effects on systemic energy homeostasis.

\section{EOSINOPHILS}

Eosinophils play an important role in helminthic and parasitic infections and allergic responses (Fulkerson and Rothenberg, 2013). Eosinophils circulate in the immature state and infiltrate and mature in specific tissues. IL-3, IL-5, and GM-CSF are required for the differentiation and activation of eosinophils. Previously, it has been demonstrated that the number of eosinophils is reduced in adipose tissue of DIO and ob/ob mice (Wu et al., 2011). In adipose tissue, eosinophils are responsible for $90 \%$ of IL-4 expression and accelerate M2 macrophage polarization by secreting Th2 type cytokines such as IL-4 and IL-13. In line with these observations, eosinophil-deficient mice display increased fat mass and inflammatory responses, as well as glucose intolerance. On the other hand, enrichment of eosinophils has beneficial effects in mice. Collectively, these data suggest that eosinophils might act as anti-inflammatory immune cells in obesity-induced adipose tissue inflammation.

\section{DENDRITIC CELLS}

Dendritic cells are the professional antigen-presenting cells that load foreign antigens onto major histocompatibility complex (MHC) molecules and present them to T lymphocytes (Hackstein and Thomson, 2004). Although it has been reported that various $\mathrm{T}$ cells are important regulators in adipose tissue inflammation, the roles of dendritic cells in adipose tissue inflammation have not been studied thoroughly. In obese mice such as DIO, ob/ob, and

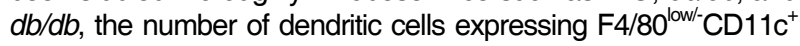
is increased in adipose tissue (Bertola et al., 2012). Furthermore, it has been reported that adipose $\mathrm{CD} 11 \mathrm{c}^{+}$cells can induce proliferation of CD4 T cells and differentiation of Th17 cells. In addition, the number of $\mathrm{CD} 11 \mathrm{c}^{+} \mathrm{CD} 1 \mathrm{c}^{+}$dendritic cells in adipose tissue shows a positive correlation with BMI. Given that dendritic cells are prominent regulators of various lymphocytes, further studies are required to investigate the role of dendritic cells in adipose tissue inflammation in obesity.

\section{T CELLS}

T cells develop and mature in the thymus, and are then repopulated into peripheral tissues. T cells have various repertoires of $T$ cell receptors and are able to discriminate self from non-self after negative and positive selection during their development in the thymus. Upon antigenic stimulation, T cells play key roles in the control of immune responses for defense against foreign antigens. There are various subpopulations of $T$ cells, including CD4, CD8, and natural killer T (NKT) cells (Jager and Kuchroo, 2010). Most subtypes of $T$ cells are involved in the regulation of adipose tissue inflammation in obesity.

The number of total T cells is increased in obese VAT in parallel with an increase in their proliferation and infiltration in response to adipose tissue-specific factors (Bornstein et al., 2000). Moreover, it has been shown that one of the T cell chemoattractant factors, RANTES, is induced in both SVCs and adipo- cytes after activation by IFN- $\gamma$ and TNF- $\alpha$. Therefore, obesityinduced factors would contribute to quantitative and qualitative changes in T cell populations, leading to the accumulation of pro-inflammatory responses in obese adipose tissues.

\section{CD4 T CELLS}

CD4 T lymphocytes recognize peptide antigens loaded on MHC class II molecules of antigen presenting cells. Naïve CD4 $T$ cells differentiate into various subtypes of CD4 T cells such as Th1, Th2, Th17, and regulatory $T$ (Treg) cells. In general, $T$ cell differentiation is regulated by a variety of cytokines, including IFN- $\gamma$ for Th1, IL-4 for Th2, IL-6 and TGF- $\beta$ for Th17, and TGF- $\beta$ for regulatory T cells (Zhu and Paul, 2008). Th1 and Th17 cells mediate pro-inflammatory responses whereas Th2 and Treg cells contribute to anti-inflammatory responses.

Winer et al. (2009) have shown that adoptive transfer of wildtype (WT) CD4 $\mathrm{T}$ cells into recombination activating gene (RAG)-null mice that lack lymphocytes results in decreased body weight gain and fat mass with improved glucose tolerance upon HFD. They suggested that Th2 cells, but not Treg cells, contribute to these beneficial effects on energy metabolism. However, unsolved questions remain with respect to the role of Th2 cells in the regulation of adipose tissue inflammation because there are no Th2-specific markers that would detect Th2 cells in adipose tissues.

Regulatory T cells, characterized as CD4 + CD25 + Foxp3 + , are a well-known anti-inflammatory $T$ cell subtype. The proportion of Treg cells among CD4 T cells is relatively high in adipose tissue compared with spleen, lymph nodes, and lung. In addition, there is a positive correlation between the proportion of Treg cells and aged adipose tissue (Feuerer et al., 2009). The number of Treg cells is decreased in adipose tissues of obese mice models such as $o b / o b, d b / d b$, and DIO relative to lean mice. Depletion of Treg cells in mice by diphtheria toxin (DT) aggravates adipose tissue inflammation and insulin resistance. On the other hand, expansion of Treg cells in mice by IL2 injection attenuates adipose tissue inflammation and improves insulin sensitivity, in part through IL-10-mediated suppression of the proliferation of conventional T cells. Notably, VAT Treg cells have adipose tissue-specific $T$ cell receptor (TCR) repertoires compared with splenic Treg cells but the identities of the antigens specific to VAT Treg cells remain to be explored. One of the distinct characteristics of Treg cells residing in VAT is a high level of PPAR $\gamma$ expression relative to Treg cells in other tissues (Cipolletta et al., 2012). Mice with PPAR $\mathrm{KO}$ specific to Foxp3-expressing cells show a significant decrease in the number of VAT Treg cells and a consequent increase in adipose tissue inflammation. On the other hand, treatment with TZD, a PPAR $\gamma$ agonist, induces an increase in VAT Treg cells followed by a reduction of inflammation in adipose tissue, indicating an important role of PPAR $\gamma$ in the accumulation and phenotype of adipose tissue Treg cells.

Th1 cells and Th17 cells are immune cell types that play critical roles in the onset of autoimmune diseases and tissue inflammatory responses. Th1 cells primarily secret IFN- $\gamma$ which stimulates monocyte differentiation into M1 type macrophages. IFN- $\gamma$ treatment of adipose tissues ex-vivo results in an increase in IP-10, MIG, and TNF- $\alpha$, implying that IFN- $\gamma$ exacerbates adipose tissue inflammation in obesity (Rocha et al., 2008). Consistent with the above observations, IFN- $\gamma$ KO mice, display improved insulin sensitivity, accompanied by a decrease in HFD-induced adipose tissue inflammation. As there are no specific markers available to distinguish adipose tissue 
Adipocytes, a Key Regulator of Adipose Tissue Inflammation

Jin Young Huh et al.

Table 1. NKT cell subsets

\begin{tabular}{|c|c|c|c|}
\hline & Type I & Type II & Type III \\
\hline Other name & Invariant NKT cells & Non-invariant NKT cells & CD1d independent NK1.1+ cells \\
\hline CD1d dependence & Yes & Yes & No \\
\hline Known antigens & $\alpha$-galactosylceramide & Sulfatide & ND \\
\hline TCR $\alpha$-chain & $\begin{array}{c}\text { V } \alpha 14 \mathrm{~J} \alpha 18 \text { (mice) } \\
\text { V } \alpha 24 \mathrm{~J} \alpha 18 \text { (humans) }\end{array}$ & Diverse & Diverse \\
\hline NK1.1(CD161) & $+/-$ & $+/-$ & + \\
\hline
\end{tabular}

*ND, not determined

resident Th1, Th2, Th17 and Treg cells, further studies are required. Nevertheless, it has been proposed that a relative decrease in anti-inflammatory cell types such as Th2 and Treg cells compared with pro-inflammatory cells such as Th1 is associated with the induction of infiltration of circulating monocytes and subsequent M1 polarization in obese adipose tissue.

\section{CD8 T CELLS}

CD8 T cells recognize peptide antigens loaded by MHC class I molecules on antigen presenting cells and participate in proinflammatory cytokine secretion and cytolysis of target cells. It has been reported that the number of CD8 T cells is elevated in obese adipose tissue. Nishimura et al. (2009) have shown that the percentage of CD8 T cells in SVCs is increased upon 2 weeks of HFD feeding whereas macrophage infiltration is induced after 6 weeks of HFD feeding. Furthermore, elevation of CD44 + CD62L (effector memory marker) CD8 T cells and a decrease in CD44-C62L + naïve CD8 T cells is observed in obese adipose tissues (Yang et al., 2010). Interestingly, the presence of CD8 T cells with a distinct TCR repertoire in obese adipose tissue has been reported, suggesting adipose tissuespecific responses of CD8 T cells in obesity. Consistent with the above observation, depletion of CD8 T cells by injection of antiCD8 antibody into DIO mice results in a decrease in the levels of pro-inflammatory cytokines such as IL- 6 and TNF- $\alpha$ with augmented glucose tolerance and insulin sensitivity independent of obesity (Nishimura et al., 2009). Moreover, in co-culture experiments of CD8 T cells with macrophages, CD8 T cells induce macrophage differentiation from monocytes and cytokine secretion, confirming the critical role of CD8 T cells in the control of macrophage polarization and activation.

\section{B CELLS}

B cells are key lymphocytes in the adaptive immune response, especially the humoral immune response. B cells not only produce antibodies but also act as antigen presenting cells. In early obesity, there is an increase in the number of immunoglobulin G (IgG)+ CD19+ B cells in VAT, indicating accumulation of class switched mature $B$ cells in obese adipose tissues (Winer et al., 2011). Also, B cell deficiency results in a reduction in VAT-resident M1 macrophages and CD8-T cell-mediated IFN- $\gamma$ expression, leading to an improvement in glucose tolerance in obesity. Additionally, transplantation of $\mathrm{MHC}$ I or MHC II molecule-deficient B cells suppresses IFN- $\gamma$ expression in both CD4 T cells and CD8 T cells in obese mice. These data indicate that $\mathrm{MHC}$ I and $\mathrm{MHC}$ II molecules on B cells would affect adipose tissue inflammation by modulating $T$ cell activity in adipose tissues. Furthermore, IgG produced by $B$ cells induces clearance of apoptotic and necrotic debris through antibody- mediated fixation of complement proteins involved in the phagocytosis of macrophages. Consequently, complement protein $\mathrm{C} 3 \mathrm{a}$ and its receptor $\mathrm{C} 3 \mathrm{aR}$ on macrophages mediate inflammation and insulin resistance. Recently, an adipose regulatory $B$ (Breg) cell population has been reported to play an intermediate anti-inflammatory role in adipose tissue inflammation via the production of IL-10 (Nishimura et al., 2013). Nevertheless, it is necessary to delineate more precisely which subtypes of $B$ cells are involved in the adipose tissue inflammation and identify the regulatory mechanisms underlying the $\mathrm{B}$ cell-mediated immune response in obese adipose tissues.

\section{NATURAL KILLER T CELLS}

Natural killer T (NKT) cells are innate lymphocytes that bridge innate and adaptive immune responses. There are three types of NKT cells, invariant NKT (iNKT, type I), non-invariant NKT (type II), and NKT-like cells (Godfrey et al., 2004). Invariant NKT and non-invariant NKT cells are CD1d-dependent, whereas NKT-like cells are CD1d-independent (Table 1). iNKT cells specifically recognize a variety of lipid antigens loaded on CD1d molecules and do not recognize peptide antigens on MHC molecules. For example, phosphatidylethanolamine, phosphatidylcholine, phosphatidylinositol, isoglobotrihexosylceramide (iGb3), $\beta$-glucosylceramide, and plasmalogen lysophosphatidylethanolamine (LPE) have been reported to be lipid antigens of CD1d (Brennan et al., 2013; Gapin et al., 2013). Among lipid antigens, $\alpha$-galactosylceramide $(\alpha-G C)$ is the most potent CD1d-binding lipid antigen for iNKT cell activation. $\alpha$-GC loaded CD1d is recognized by iNKT cells that express invariant TCR chains such as V $\alpha 14 \mathrm{~J} \alpha 18$ in mouse and V $\alpha 24 \mathrm{~J} \alpha 18$ in human. Therefore, CD1d KO mice are deficient in both type I and type II NKT cells whereas Ja18 KO mice selectively lack type I NKT cells only. To date, many studies have focused on the roles of type I NKT cells in various pathologic states.

With regard to adipose tissue inflammation in obesity, the functions of iNKT cells have received a lot of attention because iNKT cells could recognize lipid species whose amounts are dramatically increased in obesity. Although several groups have demonstrated the roles of iNKT cells in adipose tissue inflammation, the precise functions of NKT cells remain controversial. For example, Mantell et al. (2011) have suggested that the functions of iNKT cells are dispensable in adipose tissue inflammation because body weight gain, glucose sensitivity, fat mass, and adipose tissue inflammation are not significantly changed in HFD-fed CD1d KO mice. In contrast, Wu et al. (2012) have demonstrated that iNKT cells augment obesityrelated inflammation and insulin resistance in both $\mathrm{J} \alpha 18$ and CD1d KO mice although other studies using these mice have suggested an anti-inflammatory function of iNKT cells in obesity. For example, in one study HFD feeding exacerbated insulin 
Table 2. Summary of immune cells in adipose tissue

\begin{tabular}{|c|c|c|c|c|c|}
\hline Immune Cell & $\begin{array}{l}\text { \# changes } \\
\text { in obese AT }\end{array}$ & Correlation with human & $\begin{array}{c}\text { Fat mass of loss of function } \\
\text { in each immune cell }\end{array}$ & $\begin{array}{c}\text { Role of } \\
\text { adipose tissue inflammation }\end{array}$ & $\begin{array}{c}\text { Insulin } \\
\text { sensitivity }\end{array}$ \\
\hline Dendritic cell & $\uparrow$ & Yes & ND & ND & ND \\
\hline Neutrophil & $\uparrow$ & ND & $\uparrow$ & Pro-inflammatory & $\downarrow$ \\
\hline Mast Cell & $\uparrow$ & Yes & $\downarrow$ & Pro-inflammatory & $\downarrow$ \\
\hline Eosinophil & $\downarrow$ & ND & $\uparrow$ & Anti-inflammatory & $\uparrow$ \\
\hline $\mathrm{B}$ cell & $\uparrow$ & ND & $\downarrow$ (VAT:SAT ratio) & Pro-inflammatory & $\downarrow$ \\
\hline CD8 $+\mathrm{T}$ cell & $\uparrow$ & ND & $\leftrightarrow$ & Pro-inflammatory & $\downarrow$ \\
\hline CD4 + Th1 & $\uparrow$ & ND & ND & Pro-inflammatory & $\downarrow$ \\
\hline CD4 + Treg & $\downarrow$ & Yes & ND & Anti-inflammatory & $\uparrow$ \\
\hline
\end{tabular}

$\uparrow$, increased; $\downarrow$, decreased; $\leftrightarrow$, not changed; ND, not determined

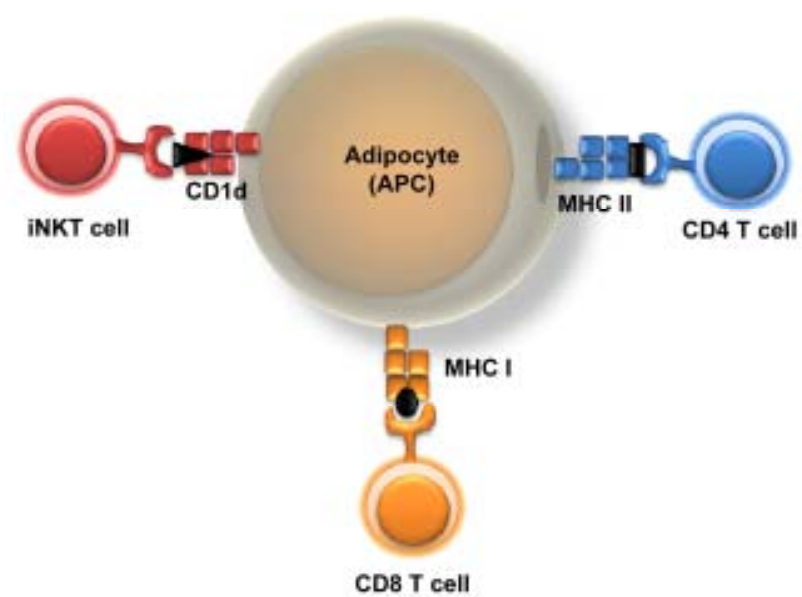

Fig. 2. Model of adipocytes as antigen presenting cells. Adipocytes could act as antigen presenting cells via expression of key molecules for antigen presentation in obese adipose tissue. Adipocytes expressing $\mathrm{MHC}$ I could mediate CD8 $\mathrm{T}$ cell responses whereas those expressing MHC II molecules could regulate CD4 T cell responses. In addition, adipocytes could modulate the function and activation of iNKT cells via high expression of CD1d molecules in adipose tissue.

resistance accompanied by increase in body weight, fat mass, and adipose tissue inflammation in both Ja18 KO and CD1d KO mice compared with WT mice (Huh et al., 2013; Lynch et al., 2012). Moreover, adoptive transfer of iNKT cells into obese mice induces loss of body weight, improved glucose tolerance, and decreased adipose tissue inflammation (Lynch et al., 2012). In addition, single or double injections of $\alpha-G C$ are sufficient to induce expression of arginase-1, which is one of the M2 marker genes, and improve insulin sensitivity (Ji et al., 2012).

\section{ADIPOCYTES AS ANTIGEN PRESENTING CELLS IN ADIPOSE TISSUE INFLAMMATION}

Traditionally, the major functions of adipocytes are to store excess energy, to protect vital organs, and to insulate the body against heat loss. However, accumulating evidence suggests that adipocytes are also endocrine cells that secrete a variety of adipokines such as leptin, adiponectin, and resistin (Ouchi et al., 2011). In obesity, adipocytes secrete pro-inflammatory cyto- kines including TNF- $\alpha$ and IL- 6 and stimulate adipose tissue inflammation. Recently, it has been suggested that adipocytes could act as antigen presenting cells to T cells in adipose tissue inflammation. Although insulin stimulates translocation of MHC class I molecules from the endoplasmic reticulum (ER) to the plasma membrane in rat brown adipocytes, there is no direct evidence for an interaction between adipocytes and CD8 T cells (Malide et al., 2001). Very recently, it has been reported that adipocytes also express MHC class II molecules and costimulatory signal molecules such as CD80 and CD86 (Deng et al., 2013). MHC class II molecules on adipocytes can functionally activate CD4 T cells in an antigen-specific and contactdependent manner. Despite these findings, the contribution of adipocyte-induced activation of $T$ cells in adipose tissue inflammation has not been clarified. Morris et al. (2013) have suggested that MHC class II molecules on macrophages, but not on adipocytes, could play critical roles in CD4 T cell activation in adipose tissue. Therefore, it would be critical to investigate the significance of adipocytes as antigen presenting cells in adipose tissue inflammation (Fig. 2).

In adipose tissue, CD1d, an antigen-presenting molecule that presents a lipid antigen, is highly expressed in adipocytes relative to SVCs composed of various immune cells (Huh et al., 2013; Schipper et al., 2012). Recently, it has been reported that $\gamma \delta \mathrm{T}$ cells can recognize and respond to CD1d molecules on antigen presenting cells (Uldrich et al., 2013). To date, a few endogenous antigens of CD1d have been demonstrated. For example, plasmalogen LPE, iGb3, and $\beta$-glucosylceramide are potential endogenous lipid antigen species that can bind to CD1d and induce subsequent activation of iNKT cells (Brennan et al., 2013). However, the specific biologic pathways mediating lipid antigen-induced activation of iNKT cells in obesity are unknown. Thus, it is of particular interest to investigate whether adipocytes actively modulate iNKT activation through presentation of lipid antigens in addition to secretion of various cytokines. Huh et al. (2013) have demonstrated that CD1d expression is reduced in obese adipose tissue, which could account for the decrease in iNKT cell number upon HFD feeding. Although the role of adipocyte CD1d in antigen presentation seems less clear cut, several characteristics that are shared between adipocytes and macrophages suggest the potential activation of iNKT cells through adipocyte CD1d-mediated antigen presentation. For example, both macrophages and adipocytes can take up and store lipids in response to nutrient cues. Furthermore, preadipocytes appear to engage in phagocytic and antimicrobial activity (Shoelson et al., 2006). Therefore, it is likely that 
HFD-induced dynamic changes in lipid metabolites loaded onto adipocyte CD1d could mediate functional alterations of iNKT cells in obesity.

\section{CONCLUSION}

In obesity, it has been suggested that inflammation in adipose tissue plays a critical role in mediating insulin resistance. In addition, various innate and adaptive immune cells in adipose tissue are apparently involved in the regulation of adipose tissue inflammation and insulin resistance (Table 2). For example, cells that secrete Th2 type cytokines such as M2 macrophages, eosinophils, and Tregs maintain insulin sensitivity in lean adipose tissue. In contrast, cells that secrete Th1-type cytokines, including M1 macrophages, Th1 cells, CD8 T cells, and mast cells, are dominant in obese adipose tissue and augment proinflammatory responses and insulin resistance (Fig. 1). Notably, adipocytes seem to act as key regulatory cells in the control of adipose tissue inflammation through cytokine secretion and antigen presentation (Fig. 2). However, there are many unanswered questions regarding the molecular mechanisms of adipose tissue inflammation and metabolism dysregulation in obesity, including the following:

1) Which cells are involved in the initiation of adipose tissue inflammation?

2) How do adipocytes and various immune cells communicate and regulate their functions?

3) What are the underlying mechanisms of inflammationinduced adipose tissue dysfunction in obesity?

4) How does adipose tissue inflammation modulate adipose tissue mass?

5) What is the molecular nature of antigens associated with adipose tissue inflammation?

When we can answer the above questions we will be closer to developing proper therapeutic targets for the treatment of obesity.

\section{ACKNOWLEDGMENTS}

This review was supported by grants from the Korea Science and Engineering Foundation funded by the Korean government [Ministry of Education, Science and Technology (MEST)] (2011-0018312). The authors have no conflict of interest to declare.

\section{REFERENCES}

Amano, S.U., Cohen, J.L., Vangala, P., Tencerova, M., Nicoloro, S.M., Yawe, J.C., Shen, Y., Czech, M.P., and Aouadi, M. (2014) Local proliferation of macrophages contributes to obesityassociated adipose tissue inflammation. Cell Metab. 19, 162 171.

Bertola, A., Ciucci, T., Rousseau, D., Bourlier, V., Duffaut, C., Bonnafous, S., Blin-Wakkach, C., Anty, R., lannelli, A., Gugenheim, J., et al. (2012). Identification of adipose tissue dendritic cells correlated with obesity-associated insulin-resistance and inducing Th17 responses in mice and patients. Diabetes 61, 22382247.

Bischoff, S.C. (2007). Role of mast cells in allergic and non-allergic immune responses: comparison of human and murine data. Nat. Rev. Immunol. 7, 93-104.

Bornstein, S.R., Abu-Asab, M., Glasow, A., Path, G., Hauner, H., Tsokos, M., Chrousos, G.P., and Scherbaum, W.A. (2000). Immunohistochemical and ultrastructural localization of leptin and leptin receptor in human white adipose tissue and differentiating human adipose cells in primary culture. Diabetes 49, 532-538.

Brennan, P.J., Brigl, M., and Brenner, M.B. (2013). Invariant natural killer T cells: an innate activation scheme linked to diverse effector functions. Nat. Rev. Immunol. 13, 101-117.

Chawla, A., Nguyen, K.D., and Goh, Y.P. (2011). Macrophagemediated inflammation in metabolic disease. Nat. Rev. Immunol. 11, 738-749.

Cipolletta, D., Feuerer, M., Li, A., Kamei, N., Lee, J., Shoelson, S.E., Benoist, C., and Mathis, D. (2012). PPAR-gamma is a major driver of the accumulation and phenotype of adipose tissue Treg cells. Nature 486, 549-553

Deng, T., Lyon, C.J., Minze, L.J., Lin, J., Zou, J., Liu, J.Z., Ren, Y., Yin, Z., Hamilton, D.J., Reardon, P.R., et al. (2013). Class II major histocompatibility complex plays an essential role in obesityinduced adipose inflammation. Cell Metab. 17, 411-422.

Feuerer, M., Herrero, L., Cipolletta, D., Naaz, A., Wong, J., Nayer, A., Lee, J., Goldfine, A.B., Benoist, C., Shoelson, S., et al. (2009). Lean, but not obese, fat is enriched for a unique population of regulatory $T$ cells that affect metabolic parameters. Nat. Med. 15, 930-939.

Fulkerson, P.C., and Rothenberg, M.E. (2013). Targeting eosinophils in allergy, inflammation and beyond. Nat. Rev. Drug Dis. 12, 117-129.

Gapin, L., Godfrey, D.I., and Rossjohn, J. (2013). Natural killer T cell obsession with self-antigens. Curr. Opin. Immunol. 25, 168173.

Godfrey, D.I., MacDonald, H.R., Kronenberg, M., Smyth, M.J., and Van Kaer, L. (2004). NKT cells: what's in a name? Nat. Rev. Immunol. 4, 231-237.

Hackstein, H., and Thomson, A.W. (2004). Dendritic cells: emerging pharmacological targets of immunosuppressive drugs. Nat. Rev. Immunol. 4, 24-34.

Ham, M., Lee, J.W., Choi, A.H., Jang, H., Choi, G., Park, J., Kozuka, C., Sears, D.D., Masuzaki, H., and Kim, J.B. (2013). Macrophage glucose-6-phosphate dehydrogenase stimulates proinflammatory responses with oxidative stress. Mol. Cell. Biol. 33, 2425-2435.

Hotamisligil, G.S., Shargill, N.S., and Spiegelman, B.M. (1993). Adipose expression of tumor necrosis factor-alpha: direct role in obesity-linked insulin resistance. Science 259, 87-91.

Huh, J.Y., Kim, J.I., Park, Y.J., Hwang, I.J., Lee, Y.S., Sohn, J.H., Lee, S.K., Alfadda, A.A., Kim, S.S., Choi, S.H., et al. (2013). A novel function of adipocytes in lipid antigen presentation to iNKT cells. Mol. Cell. Biol. 33, 328-339.

Jager, A., and Kuchroo, V.K. (2010). Effector and regulatory T-cell subsets in autoimmunity and tissue inflammation. Scand. J. Immunol. 72, 173-184.

Ji, Y., Sun, S., Xia, S., Yang, L., Li, X., and Qi, L. (2012). Short-term high-fat-diet challenge promotes alternative macrophage polarization in adipose tissue via natural killer T cells and interleukin-4. J. Biol. Chem. 287, 24378-24386.

Lee, Y.S., Li, P., Huh, J.Y., Hwang, I.J., Lu, M., Kim, J.I., Ham, M., Talukdar, S., Chen, A., Lu, W.J., et al. (2011). Inflammation is necessary for long-term but not short-term high-fat diet-induced insulin resistance. Diabetes $60,2474-2483$.

Liu, J., Divoux, A., Sun, J., Zhang, J., Clement, K., Glickman, J.N. Sukhova, G.K., Wolters, P.J., Du, J., Gorgun, C.Z., et al. (2009). Genetic deficiency and pharmacological stabilization of mast cells reduce diet-induced obesity and diabetes in mice. Nat. Med. $15,940-945$

Lumeng, C.N., Bodzin, J.L., and Saltiel, A.R. (2007a). Obesity induces a phenotypic switch in adipose tissue macrophage polarization. J. Clin. Invest. 117, 175-184.

Lumeng, C.N., Deyoung, S.M., Bodzin, J.L., and Saltiel, A.R. (2007b). Increased inflammatory properties of adipose tissue macrophages recruited during diet-induced obesity. Diabetes 56 , 16-23.

Lynch, L., Nowak, M., Varghese, B., Clark, J., Hogan, A.E., Toxavidis, V., Balk, S.P., O'Shea, D., O'Farrelly, C., and Exley, M.A (2012). Adipose tissue invariant NKT cells protect against dietinduced obesity and metabolic disorder through regulatory cytokine production. Immunity $37,574-587$.

Malide, D., Yewdell, J.W., Bennink, J.R., and Cushman, S.W. (2001). The export of major histocompatibility complex class molecules from the endoplasmic reticulum of rat brown adipose cells is acutely stimulated by insulin. Mol. Biol. Cell 12, 101-114.

Mantell, B.S., Stefanovic-Racic, M., Yang, X., Dedousis, N., Sipula I.J., and O'Doherty, R.M. (2011). Mice lacking NKT cells but with 
a complete complement of CD8+ T-cells are not protected against the metabolic abnormalities of diet-induced obesity. PLoS One 6, e19831.

Mathis, D. (2013). Immunological goings-on in visceral adipose tissue. Cell Metab. 17, 851-859.

Moller, D.E., and Kaufman, K.D. (2005). Metabolic syndrome: a clinical and molecular perspective. Annu. Rev. Med. 56, 45-62.

Morris, D.L., Cho, K.W., Delproposto, J.L., Oatmen, K.E., Geletka, L.M., Martinez-Santibanez, G., Singer, K., and Lumeng, C.N. (2013). Adipose tissue macrophages function as antigenpresenting cells and regulate adipose tissue CD4+ $\mathrm{T}$ cells in mice. Diabetes 62, 2762-2772.

Nijhuis, J., Rensen, S.S., Slaats, Y., van Dielen, F.M., Buurman, W.A., and Greve, J.W. (2009). Neutrophil activation in morbid obesity, chronic activation of acute inflammation. Obesity (Silver Spring) 17, 2014-2018.

Nishimura, S., Manabe, I., Nagasaki, M., Eto, K., Yamashita, H., Ohsugi, M., Otsu, M., Hara, K., Ueki, K., Sugiura, S., et al. (2009). CD8+ effector T cells contribute to macrophage recruitment and adipose tissue inflammation in obesity. Nat. Med. 15, 914-920.

Nishimura, S., Manabe, I., Takaki, S., Nagasaki, M., Otsu, M., Yamashita, H., Sugita, J., Yoshimura, K., Eto, K., Komuro, I., et al. (2013). Adipose natural regulatory B cells negatively control adipose tissue inflammation. Cell Metab. pii: S1550-4131(13)0 0386-0.

Ouchi, N., Parker, J.L., Lugus, J.J., and Walsh, K. (2011). Adipokines in inflammation and metabolic disease. Nat. Rev. Immunol. 11, 85-97.

Patsouris, D., Li, P.P., Thapar, D., Chapman, J., Olefsky, J.M., and Neels, J.G. (2008). Ablation of CD11c-positive cells normalizes insulin sensitivity in obese insulin resistant animals. Cell Metab. 8, 301-309.

Pham, C.T. (2006). Neutrophil serine proteases: specific regulators of inflammation. Nat. Rev. Immunol. 6, 541-550.

Rocha, V.Z., Folco, E.J., Sukhova, G., Shimizu, K., Gotsman, I. Vernon, A.H., and Libby, P. (2008). Interferon-gamma, a Th1 cytokine, regulates fat inflammation: a role for adaptive immunity in obesity. Circ. Res. 103, 467-476.

Schipper, H.S., Rakhshandehroo, M., van de Graaf, S.F., Venken, K., Koppen, A., Stienstra, R., Prop, S., Meerding, J., Hamers, N. Besra, G., et al. (2012). Natural killer T cells in adipose tissue prevent insulin resistance. J. Clin. Invest. 122, 3343-3354.

Shoelson, S.E., Lee, J., and Goldfine, A.B. (2006). Inflammation and insulin resistance. J. Clin. Invest. 116, 1793-1801.
Talukdar, S., Oh da, Y., Bandyopadhyay, G., Li, D., Xu, J., McNelis J., Lu, M., Li, P., Yan, Q., Zhu, Y., et al. (2012). Neutrophils mediate insulin resistance in mice fed a high-fat diet through secreted elastase. Nat. Med. 18, 1407-1412.

Uldrich, A.P., Le Nours, J., Pellicci, D.G., Gherardin, N.A., McPherson, K.G., Lim, R.T., Patel, O., Beddoe, T., Gras, S., Rossjohn, J., et al. (2013). CD1d-lipid antigen recognition by the gammadelta TCR. Nat. Immunol. 14, 1137-1145.

Weisberg, S.P., McCann, D., Desai, M., Rosenbaum, M., Leibel, R.L., and Ferrante, A.W., Jr. (2003). Obesity is associated with macrophage accumulation in adipose tissue. J. Clin. Invest. 112, 1796-1808.

Winer, S., Chan, Y., Paltser, G., Truong, D., Tsui, H., Bahrami, J., Dorfman, R., Wang, Y., Zielenski, J., Mastronardi, F., et al. (2009). Normalization of obesity-associated insulin resistance through immunotherapy. Nat. Med. 15, 921-929.

Winer, D.A., Winer, S., Shen, L., Wadia, P.P., Yantha, J., Paltser, G., Tsui, H., Wu, P., Davidson, M.G., Alonso, M.N., et al. (2011) $B$ cells promote insulin resistance through modulation of $T$ cells and production of pathogenic IgG antibodies. Nat. Med. 17, 610617.

Wu, D., Molofsky, A.B., Liang, H.E., Ricardo-Gonzalez, R.R., Jouihan, H.A., Bando, J.K., Chawla, A., and Locksley, R.M. (2011). Eosinophils sustain adipose alternatively activated macrophages associated with glucose homeostasis. Science 332, 243-247.

Wu, L., Parekh, V.V., Gabriel, C.L., Bracy, D.P., Marks-Shulman, P.A., Tamboli, R.A., Kim, S., Mendez-Fernandez, Y.V., Besra, G.S., Lomenick, J.P., et al. (2012). Activation of invariant natural killer T cells by lipid excess promotes tissue inflammation, insulin resistance, and hepatic steatosis in obese mice. Proc. Natl. Acad. Sci. USA 109, E1143-1152.

Xu, H., Barnes, G.T., Yang, Q., Tan, G., Yang, D., Chou, C.J., Sole, J., Nichols, A., Ross, J.S., Tartaglia, L.A., et al. (2003). Chronic inflammation in fat plays a crucial role in the development of obesity-related insulin resistance. J. Clin. Invest. 112, 1821-1830.

Yang, H., Youm, Y.H., Vandanmagsar, B., Ravussin, A., Gimble, J.M., Greenway, F., Stephens, J.M., Mynatt, R.L., and Dixit, V.D. (2010). Obesity increases the production of proinflammatory mediators from adipose tissue T cells and compromises TCR repertoire diversity: implications for systemic inflammation and insulin resistance. J. Immunol. 185, 1836-1845.

Zhu, J., and Paul, W.E. (2008). CD4 T cells: fates, functions, and faults. Blood 112, 1557-1569. 